\title{
A Review of High Energy Polarimetry, With a view toward RHIC
}

\author{
David G. Underwood \\ High Energy Physics \\ Argonne National Laboratory \\ Argonne II 60439
}

\begin{abstract}
We present four physics processes that have measured asymmetries at high energy. Some problems and some advantages of utilizing each in a polarimeter are discussed. For each we give references to examples of experimental setup and data.

INTRODUCTION

The energy dependence of the analyzing power for the processes we discuss here is shown in a general way in reference 1 . The analyzing power from interference of strong amplitudes in elastic or quasi-elastic scattering falls as roughly the inverse of the beam momentum. The others, Inverse-Primakoff, Coulomb-Nuclear, and large-x pion inclusive appear to be energy independent in the relevant ranges.

The possibilities can be evaluated with the following parameters in mind:

+ Analyzing power vs Energy,

+ Usable Luminosity,

+ Quality factor, $\mathrm{A}^{2} \mathrm{~N}$,

+ The continuum from Online Scaler readout to Difficult analysis,

+ Absolute vs Relative Analyzing power,

+ Internal to an Accelerator vs in External Beamline.
\end{abstract}

These parameters are discussed in terms of the advantages and difficulties for each polarimeter.

\section{P P Elastic and P N elastic}

There are a number of problems which make elastic scattering a more difficult process to utilize at increasingly high energy. The analyzing power typically peaks near a $t$, momentum transfer squared, of $0.15(\mathrm{GeV} / \mathrm{c})^{2}$, and falls off at higher $\mathrm{t}$. The analyzing power falls roughly as $1 / \mathrm{p}$ lab, so that at $100 \mathrm{GeV} / \mathrm{c}$, the analyzing power is down to about $1 \%$. The next problem is that the increasing number of pions produced at high 


\section{DISCLAIMER}

This report was prepared as an account of work sponsored by an agency of the United States Government. Neither the United States Government nor any agency thereof, nor any of their employees, makes any warranty, express or implied, or assumes any legal liability or responsibility for the accuracy, completeness, or usefulness of any information, apparatus, product, or process disclosed, or represents that its use would not infringe privately owned rights. Reference herein to any specific commercial product, process, or service by trade name, trademark, manufacturer, or otherwise does not necessarily constitute or imply its endorsement, recommendation, or favoring by the United States Government or any agency thereof. The views and opinions of authors expressed herein do not necessarily state or reflect those of the United States Government or any agency thereof. 


\section{DISCLAIMER}

Portions of this document may be illegible in electronic image products. Images are produced from the best available original document. 
energy is a serious background to both the forward and the recoil detection. The angular separation between the forward scatter and the beam becomes small so that, for example, at $300 \mathrm{GeV}$ in Fermilab experiment 61, a septum was required in a Cherenkov counter at 100 meters from the target. 2 One way around these difficulties which was used in an AGS external beamline up to $22 \mathrm{GeV}$ was to utilize magnetic spectrometers for the forward and recioil arms, and to accept somewhat larger angle forward scatters and higher $t$ at the highest energies. ${ }^{3}$

Elastic scattering has been used effectively for internal polarimeters in accelerators even in the face of difficulties like limited space and a quasi-elastic background from $\mathrm{CH}$ targets. One example is at KEK for energies up to $12 \mathrm{GeV} .^{4}$

Another recent example is the polarimeter in the AGS for the partial Siberian snake tests. ${ }^{5}$ In this case, the forward scatter was inaccessible, both because the small forward angle put it inside the beam pipe at some energies, and because of high background rates. The recoil arms were designed to use an angle-energy correlation to separate elastics from quasi-elastics, with the protons stopping in a plastic scintillator for the energy measurement. A veto counter was used to eliminate penetrating pions. A previous version of the polarimeter utilized range information in a limited way. This version has a very great improvement, almost an order of magnitude, in eliminating the quasi elastics from carbon in the fishline target, but it is not perfect. A carbon fiber was added to the target flipping mechanism so that measurements were made with $\mathrm{C}$ and $\mathrm{CH}_{2}$ targets, and a carbon subtraction could be made from the data.

There were some interesting technical aspects to this polarimeter. The energy-angle correlation in the recoil proton measurement was done with memory lookup units which could handle several tens of millions of events per second peak rate. The new target flipping mechanism could place the target in the beam in $\mathbf{5 0}$ milliseconds while the fishline was spooling at a meter per second to reduce heat and radiation damage. $\mathrm{A}$ baler for spooling the fishline contributed to the overall reliability during the two week run.

\section{COULOMB-NUCLEAR INTERFERENCE}

The Coulomb-nuclear interference polarimeter utilizes the interference between the spin flip part of the Coulomb elastic amplitude, and the large imaginary part of the strong amplitude. From the known amplitudes in the $p$ p case, one can calculate the analyzing power and the $t$ value in elastic scattering where the maximum occurs. The maximum is 
about $4 \%$, almost independent of energy, at $-t$ of $0.005(\mathrm{GeV} / \mathrm{c})^{2}$. Calculations, and to some extent data, indicate similar parameters for $p$-Carbon elastic. ${ }^{6}$

One needs good kinematic constraints to separate the elastic scattering from diffraction dissociation, which has a similar low energy recoil and fast forward particles. A precise $t$ measurement is needed, for example +-0.001 at $t$ of $-0.003(\mathrm{GeV} / \mathrm{c})^{2}$. In the fixed target experiments done to date, primarily Fermilab experiment E704, ${ }^{7}$ the main experimental problem was to isolate and measure the recoil. For this reason, a momentum and angle measurement on the forward scattered particle was also done to help in the kinematic constraint. The forward particle is at an angle of about $1.5 \times 10^{-4}$ radian at $200 \mathrm{GeV} / \mathrm{c}$, so that it was within the beam phase space for the measurements done. A particular problem with a solid sensitive target is that the beam particles deposit energy in the target with magnitude comparable to that deposited by the recoil. For some of the Fermilab data, a Stilbene target was used, which has a different scintillation pulse time dependence for fast minimum ionizing particles, such as the beam, than for slow stopping protons, such as the recoil.

There is some interest in using this process inside an accelerator with a gas jet target. for example at RHIC. The kinematics of the forward particle are then the same as in the unpolarized experiments to measure the real part of the elastic amplitude. These experiments use Roman pot detectors in the accelerator lattice. The recoil particle detecton would be free of carbon background and energy deposition by the beam. However, precise measurements of the 30 to $100 \mathrm{MeV} / \mathrm{c}$ momentum recoil may be difficult in an environment with many pions. Many pions are produced each $110 \mathrm{~ns}$ when a beam bunch crosses the target. There are also beam-gas backgrounds from the accelerator.

It should also be possible to use the Coulomb -nuclear method with elastic scattering of the two proton beams in a collider, with Roman pots on either side of the interaction region. One has to consider the reduced luminosity possible at the high beta parameter needed to detect the scatters, in light of the number of events needed at the low analyzing power. High beta means the beam is almost parallel trajectories and not tightly focused.

\section{INVERSE PRIMAKOFF}

Primakoff originally proposed using the connection between S-channel and T-channel scattering as a way to measure the electromagnetic coupling, radiative width, of 
short lived particles. This is of interest to us because we can relate a body of low energy data on polarized photoproduction of baryon resonances to the high energy process of producing these resonances in the Coulomb field of a nucleus. For example, the asymmetry in the Coulomb dissociation of high energy protons into a proton plus pizero with invariant mass near $1350 \mathrm{MeV} / \mathrm{c}^{2}$ is directly related to the asymmetry in photoproduction on a polarized proton target with photons of 400 to $500 \mathrm{MeV}$. This has been done at $200 \mathrm{GeV}$ in Fermilab Experiment E704, and it works, albeit with some experimental difficulties. 7,8

The primary experimental difficulty in this type of polarimeter is the separation of Coulomb dissociation from diffraction dissociation. The parameter which is used for this separation is the slope of the t distribution. For Coulomb events the distribution has a slope determined primarily by experimental resolution, which has to be about $\mathrm{e}-2000 \mathrm{t}$ in order to do the separation. The slope for strong diffraction is approximately $e-10 A^{2 / 3 t}$, which is $e-400 t$ for diffraction off a lead nucleus. The direction of the proton-pi-zero effective mass must be determined to about $+-3 \times 10^{-5}$ radian at 200 $\mathrm{GeV} / \mathrm{c}$. This requires a rather good spectrometer for the proton and a photon detector with good position and energy resolution for the pi-zero.

In principle, an analyzing power of $90 \%$ is possible independent of energy. In practice the analyzing power is dominated by the fraction of background, which can be determined well, but not eliminated. Thus, in E704, the measured analyzing power was about $30 \%$, and substantial analysis effort was required, at the level of a PhD thesis. This may be practical for a one-time absolute polarization measurement, but not for a beam tuning polarimeter where information is required quickly and repeatedly.

\section{PION INCLUSIVE}

Large asymmetries in the production of very forward pions from polarized proton beams have been observed over a large range of energy. There are data at 6 and 12 $\mathrm{GeV}$ from the ZGS, and at $200 \mathrm{GeV}$ in E704. ${ }^{9}$ There are also pion production asymmetries with polarized targets at Cern at $30 \mathrm{GeV}$ and at Serpukhov at $70 \mathrm{GeV}$. The $200 \mathrm{GeV}$ data are well characterized in terms of $x f$ and $\mathrm{pt}$, and will be useful at similar energies. One can speculate that the effects are largely energy independent, however there is insufficient data to connect all the energies in a useful parameterization to show this. As shown in reference 9, the pi + and pi - asymmetries are large, almost linear in $\mathbf{x f}$, and approximately mirror images of each other. The pi-zero asymmetry is smaller. 
The RHIC Spin Collaboration is considering this process for an intemal polarimeter in the RHIC machine. Measurements are desired on a repetative basis several times per hour. $\mathrm{Pi}$ - production appears to have several advantages. $\mathrm{Pi}+$ would require the separation of protons at forward angle and high momentum whereas the anti proton flux in this region is negligible. The Kaon flux could either be considered a dilution factor or could possibly be eliminated with a Cherenkov. Pi-zeros would require something like a lead glass array very close to the beam pipe and considerable event reconstruction effort.

The optimum pion momentum has been determined by finding the derivative of a parameterization for $A^{2} N$, the quality factor which minimizes error bars. The cross secton was parameterized as a function of $x f$ above pt of $0.7 \mathrm{Gev} / \mathrm{c}$ and the asymmetry was also so parameterized. The result is that $x f$ of about $1 / 2$ is optimum. The apparatus must select pions of $1 / 2$ the proton momentum and pt of greater than $0.7 \mathrm{GeV} / \mathrm{c}$. This may be done with magnetic spectrometers using either limited aperture or very fast electronics to correlate hodoscopes, so that primarily events of interest are scaled.

\section{References}

1) Summary of Polarimeter Session, D. G. Underwood, High Energy Spin Physics, AIP Conf. Proc. 187, Particles and Fields 37, 1352 (1988)

2) R.V. Kline et al. Phys. Rev. D 22553 (1980)

3) F. Khiari et al. Univ of Michigan Report UM-HE-87-36 (1988)

4) Internal Polarimeters for the Polarized Proton Beam at the KEK PS, H. Sato et al. High Energy Spin Physics, AIP Conf. Proc. 187, Particles and Fields 37, 1355 (1988)

5) Preservation of Proton Polarization by a Partial Snake, $H$. Huang et al. to be published Phys Rev Lett Nov, 1994

6) A. K. Akchurin et al. Phys Rev D 48, 3026 (1993)

7) D. Grosnick et al. NIM A 290, 269 (1990)

8) D. C. Carey et al. Phys Rev Lett 64, 358 (1990)

9) D. L. Adams et al. Phys Lett B 264, 14 (1991)

* Work supported by the U.S. Department of Energy, Division of High Energy Physics, Contract W-31-109-ENG-38. 